\title{
Economic evaluation of mindfulness group therapy for patients with depression, anxiety, stress and adjustment disorders compared with treatment as usual
}

\author{
Sanjib Saha, Johan Jarl, Ulf-G Gerdtham, Kristina Sundquist and Jan Sundquist
}

\section{Background}

A randomised controlled trial found that a structured mindfulness group therapy (MGT) programme was as effective as treatment as usual (mostly cognitive-behavioural therapy) for patients with a diagnosis of depression, anxiety or stress and adjustment disorders in Sweden (ClinicalTrials.gov: NCT01476371).

\section{Aims}

To perform a cost-effectiveness analysis of MGT compared with treatment as usual from both a healthcare and a societal perspective for the trial duration (8 weeks).

\section{Method}

The costs from a healthcare perspective included treatment as usual, medication and costs for providing MGT. The societal perspective included costs from the healthcare perspective plus savings from productivity gains for the trial duration. The effectiveness was measured as quality-adjusted life-years (QALY) using the EQ-5D-5L questionnaire and the UK value set. Uncertainty surrounding the incremental costs and effects were estimated using non-parametric bootstrapping with 5000 replications and presented with $95 \%$ confidence intervals and costeffectiveness acceptability curves.

\section{Results}

The MGT group had significantly lower healthcare and societal costs (mean differences $€ 115(95 \% \mathrm{Cl}-193$ to -36$)$ and $-€ 112$ (95\% Cl -207 to -17$)$, respectively) compared with the control group. In terms of effectiveness, there was no significant difference in QALY gain (mean difference $-0.003,95 \% \mathrm{Cl}-0.0076$ to 0.0012) between the two groups.

\section{Conclusions}

MGT is a cost-saving alternative to treatment as usual over the trial duration from both a healthcare and a societal perspective for patients with a diagnosis of depression, anxiety or stress and adjustment disorders in Sweden.

\section{Declaration of interest}

None.

\section{Keywords}

Economic evaluation; mindfulness group therapy; cognitive behavioural therapy.

\section{Copyright and usage}

(c) The Royal College of Psychiatrists 2018.
The standard treatment for depression, anxiety, stress and adjustment disorders includes pharmacological treatment and/or different types of psychotherapy. In Swedish general practice, cognitive-behavioural therapy (CBT), a form of psychotherapy, has been introduced as a standard treatment for depressive disorders However, the individual therapeutic approach requires a considerable number of CBT therapists who are in short supply, which results in limited access and long waiting times for patients. Moreover, an individualist approach is resource intensive and thus results in high costs for the healthcare system. An alternative to the individualist approach is mindfulness-based therapies that are accessible to a large group of patients. After being introduced to the therapy, patients can practise mindfulness on their own. ${ }^{2}$

Considering these advantages of mindfulness-based therapy, a randomised controlled trial (RCT) of a structured mindfulness group therapy (MGT) programme was conducted comparing it with treatment as usual (TAU) (mostly CBT) in patients with a diagnosis of depression, anxiety, stress and adjustment disorders in southern Sweden (ClinicalTrials.gov: NCT01476371). The trial showed that MGT was not inferior to TAU in terms of scores on the Montgomery-Åsberg Depression Rating Scale (self-rated version) (MADRS-S), Hospital Anxiety and Depression Scale (HADS, anxiety and depression subscales) and Patient Health Questionnaire-9 (PHQ-9) ${ }^{3}$ and a broad range of psychiatric symptoms including the Symptom Checklist-90 and Mindful Attention Awareness Scale. ${ }^{4}$ This raises the question of whether MGT, being as effective as TAU, is a better use of society's scarce resources (i.e. whether it is cost saving). The existing published evidence is inconclusive on the cost-effectiveness of different mindfulnessbased group therapy interventions. ${ }^{5-7}$ Therefore, the objective of the current study was to perform an economic evaluation of the above-mentioned trial comparing MGT with TAU from both a societal and a healthcare perspective in Sweden.

\section{Method}

\section{The trial}

The RCT was conducted in Scania county of southern Sweden. A total of 24 out of 150 primary healthcare centres (PHCs) were randomly chosen to be included in the trial. Out of these 24 PHCs, 16 agreed to participate. Patient enrolment commenced on 4 January 2012 and ended on 22 March 2012. Newly diagnosed patients with depression, anxiety or stress and adjustment disorders were eligible together with individuals who already had a history of these disorders. Details of the patient recruitment, inclusion and exclusion criteria can be found in supplementary Appendix 1 available at https://doi.org/10. 1192/bjp.2018.247 and in other publications on this RCT., ${ }^{3,4}$ A total of 215 patients were randomised into the intervention group $(n=110)$ and the control group $(n=105)$. The characteristics of the participants at baseline and follow-up are presented in Table 1.

\section{Intervention - MGT}

The intervention was based on two well-established mindfulness-based therapies: mindfulness-based stress reduction and mindfulness-based 
Table 1 Characteristics of the participants at baseline and 8-week follow-up

\begin{tabular}{|c|c|c|c|c|c|c|c|c|c|c|c|c|c|c|c|c|}
\hline & \multicolumn{8}{|c|}{ Baseline } & \multicolumn{8}{|c|}{ Follow-up (8-week) } \\
\hline & \multicolumn{4}{|c|}{ Mindfulness group } & \multicolumn{4}{|c|}{ Control group } & \multicolumn{3}{|c|}{ Mindfulness group } & \multicolumn{5}{|c|}{ Control group } \\
\hline & Mean & s.d. & $n$ & $\%$ & Mean & s.d. & $n$ & $\%$ & Mean & s.d. & $n$ & $\%$ & Mean & s.d. & $n$ & $\%$ \\
\hline Age & 42.00 & 10.94 & 110 & & 41.00 & 11.00 & 105 & & 42.20 & 10.81 & 90 & & 40.63 & 11.75 & 84 & \\
\hline Quality-adjusted life-years & 0.662 & 0.19 & 98 & & 0.65 & 0.25 & 95 & & 0.77 & 0.17 & 87 & & 0.77 & 0.19 & 78 & \\
\hline $\begin{array}{l}\text { Employment hours per week } \\
\text { Gender }\end{array}$ & 36.17 & 7.93 & 72 & & 36.08 & 12.15 & 65 & & 35.83 & 7.7 & 58 & & 36.57 & 12.25 & 51 & \\
\hline Men & & & 20 & 18 & & & 12 & 11 & & & 13 & 14 & & & 7 & 8 \\
\hline Women & & & 90 & 82 & & & 93 & 89 & & & 77 & 86 & & & 77 & 92 \\
\hline \multicolumn{17}{|l|}{ Civil status } \\
\hline Single & & & 38 & 35 & & & 33 & 33 & & & 30 & 34 & & & 27 & 33 \\
\hline Married/living together & & & 70 & 65 & & & 67 & 67 & & & 58 & 66 & & & 56 & 67 \\
\hline \multicolumn{17}{|l|}{ Sick leave } \\
\hline No (0\%) & & & 82 & 78 & & & 78 & 74 & & & 76 & 81 & & & 74 & 76 \\
\hline Full time (100\%) & & & 18 & 17 & & & 21 & 20 & & & 10 & 11 & & & 16 & 17 \\
\hline Part time (25-75\%) & & & 5 & 5 & & & 6 & 6 & & & 8 & 8 & & & 7 & 7 \\
\hline \multicolumn{17}{|l|}{ Have a job } \\
\hline Yes & & & 72 & 65 & & & 66 & 63 & & & 59 & 66 & & & 53 & 63 \\
\hline No & & & 38 & 35 & & & 39 & 37 & & & 31 & 34 & & & 31 & 37 \\
\hline \multicolumn{17}{|l|}{ Use of antidepressant drugs } \\
\hline Yes & & & 50 & 45 & & & 36 & 34 & & & 39 & 43 & & & 30 & 37 \\
\hline No & & & 60 & 55 & & & 69 & 66 & & & 51 & 57 & & & 51 & 63 \\
\hline \multicolumn{17}{|l|}{ Use of anxiolytic drugs } \\
\hline Yes & & & 24 & 22 & & & 25 & 24 & & & 18 & 20 & & & 16 & 19 \\
\hline No & & & 86 & 78 & & & 80 & 76 & & & 72 & 80 & & & 67 & 81 \\
\hline \multicolumn{17}{|l|}{ Use of sleeping pills } \\
\hline Yes & & & 27 & 25 & & & 29 & 28 & & & 17 & 19 & & & 24 & 29 \\
\hline No & & & 83 & 75 & & & 76 & 72 & & & 73 & 81 & & & 59 & 71 \\
\hline \multicolumn{17}{|l|}{ Use of psychotherapy } \\
\hline Yes & & & 46 & 42 & & & 33 & 31 & & & 36 & 40 & & & 29 & 35 \\
\hline No & & & 64 & 58 & & & 72 & 69 & & & 54 & 60 & & & 54 & 65 \\
\hline
\end{tabular}

cognitive therapy. ${ }^{8,9}$ It included structured and controlled meditative exercises. The MGT lasted 8 weeks and was given in $2 \mathrm{~h}$ sessions on a weekly basis. Two mindfulness instructors were present at each group session and each group consisted of a maximum of ten participants. The participants were also instructed to practise mindfulness at home for $20 \mathrm{~min}$ a day and were given a compact disc of recorded mindfulness meditations, a training manual and a diary for this purpose.

\section{Control group}

The control group received healthcare for these types of conditions at their PHC, which often included pharmacological treatment, and in most cases also psychotherapy or counselling. The majority of the patients in the control group received CBT $(n=80,76 \%)$ over, on average, 6.3 individual sessions. Other therapies included, for example, basic body awareness and physical activity. There were no differences in the proportion of participants on antidepressants and tranquiliser medications between the mindfulness and control groups at baseline.

\section{Outcome measures}

We used quality-adjusted life-years (QALYs) as the only outcome measure in this study. The QALY was measured using the EQ5D-5L questionnaire ${ }^{10}$ at baseline and 8-week follow-up. The EQ$5 \mathrm{D}-5 \mathrm{~L}$ is a generic preferences-based measure of health-related quality of life with five dimensions: mobility, self-care, usual activities, pain/discomfort and anxiety/depression. Each dimension has five levels: no problems, slight problems, moderate problems, severe problems and extreme problems. These health states are translated into a score varying between 0 (equivalent of dead) and 1 (perfect health). We used the UK value set to estimate QALYs as no Swedish equivalent has yet been developed for the
5L-version. ${ }^{11}$ The QALY gain from baseline to 8 weeks was estimated by applying the area-under-the-curve ${ }^{12}$ method assuming a linear increase in the QALY between the two measurement points.

Despite the randomisation of the patients into the different forms of treatment, there was a numerical, although insignificant, difference in QALYs at baseline (Table 1); we therefore measured the effect of the intervention as gain in QALYs in the follow-up controlling for the baseline differences.

\section{Cost and service use}

The economic evaluation was conducted both from a healthcare and a societal perspective. A societal perspective includes all costs irrespective of who is burdened by them, whereas the healthcare perspective is only concerned with costs burdening the healthcare sector (although the health benefits of the patients are the effectiveness measure).$^{13}$ First, the cost for providing MGT was calculated based on the total number of hours for the mindfulness instructors providing group sessions divided by the total number of mindfulness group participants (details of the MGT cost calculation are presented in the supplementary Appendix 1). The participants reported medication use, of up to six drugs, during the 8-week trial period, including the name, strength, dosages, start date and end date. Based on this, the total medication cost per patient was calculated using unit costs from the Swedish Dental and Pharmaceutical Benefits Agency (https://www.tlv.se).

The patient questionnaire also included the number of healthcare visits separately for CBT therapist, counsellors, physiotherapists or any other therapist. Costs were calculated by multiplying the respective number of visits by standard costs according to the Swedish Association of Local Authorities and Regions database ${ }^{14}$ (mean number of visits, unit costs and sources are presented in supplementary Table 1). The cost from a healthcare perspective includes costs for TAU, medication costs and MGT costs. All 
costs were estimated in Swedish Kronor (SEK) using the 2012 price year and converted to euros (EUR) using the 2012 exchange rate of 8.705 SEK/EUR. ${ }^{15}$

\section{Productivity}

The questionnaires also contained information on participants' level of sick leave at baseline and after the 8-week trial, together with working hours per week. The productivity gain was estimated as the change in hours worked using the human capital approach, ${ }^{16}$ where each hour is valued using the gross salary (including taxes). Since participants' salary was not available from the data, we used the average monthly gross salary of $€ 3538$ (29 800 SEK) in $2012 .{ }^{17}$ This gives a value of a worked hour of $€ 23$ (186.25 SEK). Since it is unknown when the participants changed their sick leave status during the trial period, we conservatively assumed that the sick leave reported at baseline remained unchanged for the first 7 weeks, while the sick leave status reported after the trial was the status during the final (eighth) trial week.

\section{Statistical analyses}

The incremental cost-effectiveness ratio (ICER) was calculated by dividing the incremental costs by the incremental effects. The ICER represents the additional costs needed to gain one extra unit of effect, i.e. QALY in the mindfulness group compared with the control group. Uncertainty surrounding the incremental costs and effects were estimated using non-parametric bootstrapping with 5000 replications. ${ }^{18}$ The $95 \%$ confidence intervals around the mean cost differences were estimated using the approximate bootstrap confidence algorithm ${ }^{19}$ followed by Student's $t$-test. Bootstrapped incremental cost-effect pairs were plotted on a costeffectiveness plane, which shows the difference in effect on the horizontal axis and the difference in costs on the vertical axis ${ }^{20}$ of the two interventions. If all points are in the southeast or the northwest quadrant, the choice between the intervention is clear. In the southeast quadrant, the new therapy is both more effective and less costly than standard care and thus dominates standard care. The opposite is true in the northwest quadrant. In the northeast and southwest quadrants, the choice depends on the amount of money society is willing to pay to gain one unit of effect. ${ }^{13,21}$ Cost-effectiveness acceptability curves (CEACs) were also estimated to examine the probability that the intervention is cost-effective in comparison with the control group for a range of willingness-to-pay thresholds. We apply the threshold suggested by the National Institute for Health and Care Excellence in the UK of $£ 20000$ /QALY in the current study (corresponding to $€ 24691$ in 2012 year's price level) as Sweden does not have any formal threshold. ${ }^{22}$ This means that ICERs below $€ 24691$ are considered cost-effective whereas higher ICERs are not cost-effective.

The economic evaluation has been conducted following the Consolidated Health Economic Evaluation Reporting Standards (CHEERS) statement (supplementary Appendix 2). ${ }^{23}$ The statistical analyses were conducted in Stata 15.

\section{Subgroup and sensitivity analyses}

We performed several subgroup analyses to capture heterogeneity and sensitivity analyses to capture uncertainties around the findings.

(a) Subgroup analysis - gender: results are reported separately for men and women (analyses 1a, b) to capture potential heterogeneity.

(b) Subgroup analysis - sick leave: results are reported separately for the participants who were/were not on sick leave at baseline (analyses 2a, b). (c) Sensitivity analysis - missing cases: we imputed the missing data on costs and QALYs for both group using multiple imputations. ${ }^{24}$ We generated five different data-sets for each group in SPSS (version 25) using the Markov chain Monte Carlo method. Thereafter, the multiple data-sets were analysed and pooled estimates were computed following the methods described by Rubin ${ }^{25}$ (analysis 3).

(d) Sensitivity analysis - excluding outliers: $5 \%$ of the participants in both the mindfulness group and the control group with the highest healthcare cost and highest $2.5 \%$ and lowest $2.5 \%$ productivity gain costs are excluded (analyses $4 \mathrm{a}, \mathrm{b}$ ). ${ }^{26} \mathrm{~A}$ sensitivity analysis is also performed excluding the highest and lowest $2.5 \%$ in terms of QALYs gain (analysis 4c).

(e) Sensitivity analysis - participants' attendance in mindfulness sessions: to be in line with the effectiveness trial, ${ }^{3,4}$ we performed sensitivity analyses based on level of participation in the mindfulness sessions, categorised as $1-5$ and 6-8 sessions (analyses 5a, b).

\section{Ethical considerations}

The study was approved by the Ethics Committee of Lund University (application number: 2011/491). Written informed consent was obtained from all participants. All data were analysed anonymously, and the results are reported at the group level.

\section{Results}

\section{Costs and effects}

In terms of TAU costs, significant differences between the groups can be seen (Table 2). Only one of the participants in the MGT group received TAU during the trial period, leading to significantly lower cost for the MGT group (mean difference - $€ 520$, 95\% CI -583 to -456$)$. There was no statistically significant difference in the medication costs over the 8 weeks between the two groups (mean difference $€ 7,95 \% \mathrm{CI}-36$ to 51 ). The healthcare perspective included the cost of TAU, medications and providing MGT. From a healthcare perspective, the mindfulness group had significantly lower costs than the control group (mean difference $-€ 115$, $95 \%$ CI -193 to -36 ). In terms of productivity gain, there was no significant difference in mean gain between the mindfulness and the control group (mean difference $-€ 3,95 \%$ CI -66 to 60 ). From a societal perspective, the mindfulness group had significantly lower costs than the control group, (mean difference $-€ 112$, $95 \%$ CI -207 to -17 ).

In terms of effect, both groups had an insignificant increase in QALYs at the end of the 8 weeks (Table 1). Controlling for the baseline QALY differences between the two groups, there were no statistically significant differences between the mindfulness group and the control group although the latter had gained 0.003 more QALYs (95\% CI -0.0012 to 0.0076).

Thus, there were significant differences in costs both from the healthcare and societal perspective at 8 weeks in favour of the mindfulness group (Table 2) but no significant differences in QALYs; this indicates that MGT is a cost-saving intervention compared with TAU. The cost-effectiveness plane shows that almost all the incremental cost-effect pairs (bootstrapped) are located in the southwest quadrant both from the societal perspective (91\%) and the healthcare perspective (92\%) (supplementary Figs 1 and 2). Organising the bootstrapped results into a CEAC shows that the mindfulness intervention has a $67 \%$ probability of being cost-effective from the societal perspective and $70 \%$ from the healthcare perspective (Fig. 1 and supplementary Fig. 3). 
Table 2 Mean cost (in euros), effect (differences by bootstrap (5000) for control and mindfulness group) and incremental cost-effectiveness ratios ${ }^{a}$

\begin{tabular}{|c|c|c|c|}
\hline & Mindfulness group & Control group & Difference $(95 \% \mathrm{Cl})$ \\
\hline \multicolumn{4}{|l|}{ Cost, mean (s.e.) } \\
\hline Treatment as usual ${ }^{b}$ & $4(4)$ & $524(32)$ & $-520(-456$ to -583$)$ \\
\hline Medication & $43(21)$ & $36(7)$ & 7 (-36 to 51$)$ \\
\hline Mindfulness group therapy cost & 398 & 0 & $398^{\mathrm{C}}$ \\
\hline Total cost (healthcare perspective) ${ }^{b}$ & $445(21)$ & $560(34)$ & $-115(-193$ to -36$)$ \\
\hline \multicolumn{4}{|l|}{ Societal perspective } \\
\hline Productivity gain & $24(12)$ & $27(29)$ & $-3(-66$ to 60$)$ \\
\hline Total cost (societal perspective) ${ }^{b}$ & $421(25)$ & $533(41)$ & $-112(-207$ to -17$)$ \\
\hline \multicolumn{4}{|l|}{ Effect, mean (s.e.) } \\
\hline & Incremental cost-effectiveness ratios & $0.011(0.002)$ & $-0.003(-0.008$ to 0.001$)$ \\
\hline Healthcare perspective & Mindfulness is cost & & \\
\hline Societal perspective & Mindfulness is cost & & \\
\hline
\end{tabular}

\section{Subgroup and sensitivity analyses}

The results of the subgroup and sensitivity analyses are presented in Table 3. Stratifying for men, there were no significant differences in cost between treatments (analysis 1a). From the societal perspective, the results for women (analysis $1 \mathrm{~b}$ ) and when removing the outliers in QALYs gained (analysis 4c) also show no significant differences in cost. However, for all other subgroup and sensitivity analyses, the base case results are upheld.

\section{Discussion}

\section{Main findings}

We performed an economic evaluation of an MGT intervention for participants with depression, anxiety, stress and adjustment disorders from both a healthcare and a societal perspective. The intervention is cost saving compared with TAU over 8 weeks both from the healthcare and societal perspective as no significant differences in effect (i.e. QALYs) was observed.

Previous effectiveness studies of this RCT have revealed that both groups had significant improvements in health as measured by MADRS-S, HADS (anxiety and depression subscales) and PHQ-9., In contrast, we found no significant positive effect of the treatments measured in QALYs, although an insignificant increase was noted in favour of TAU. The conclusion from the effectiveness studies ${ }^{3,4}$ was that MGT is not inferior compared with TAU with the potential to save resources. From the current economic evaluation study, we can add that MGT indeed saved valuable healthcare and societal resources with equal effect as TAU over the 8-week trial period. With the current prevalence

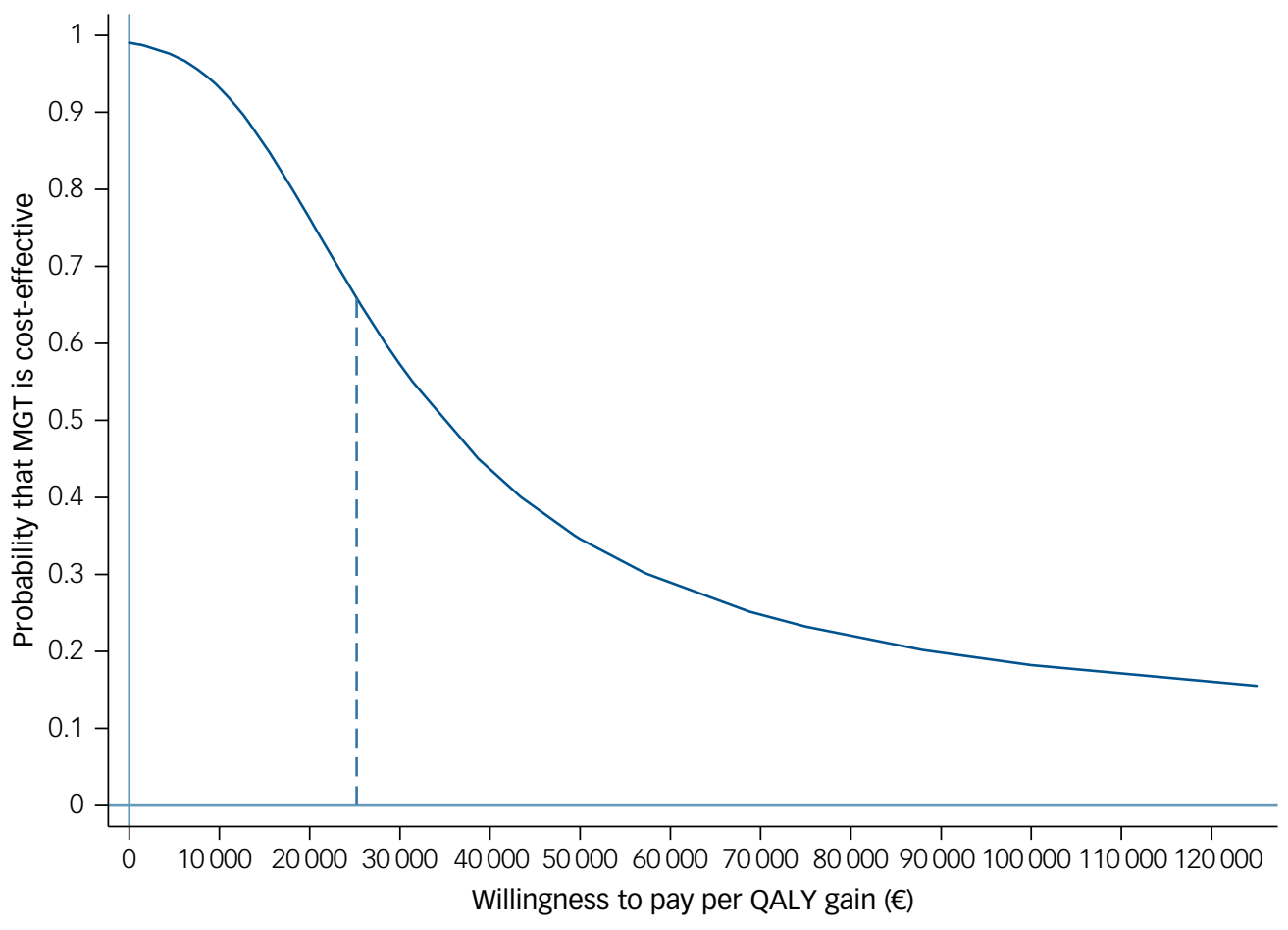

Fig. 1 Cost-effectiveness acceptability curve from the societal perspective.

Cost-effectiveness acceptability curve indicating the probability of the intervention being cost-effective at different values ( $€$ ) of willingness-to-pay per quality-adjusted life-year (QALY). The dotted line is the National Institute for Health and Care Excellence threshold applied in the current study. MGT, mindfulness group therapy. 
(2.4\%) and incidence (18.4 per 1000 person-years) of clinically diagnosed depression and anxiety disorders in Swedish PHCs, ${ }^{27}$ a societal saving of $€ 112$ per person in an 8 -week period will save a considerable amount of resources.

The bootstrapped cost-effectiveness plane, which accounts for the uncertainty in the base case estimation, shows that most of the incremental cost-effect pairs are in the southwest quadrant (supplementary Figs 1 and 2). This is because the control group, although not statistically significant, has gained slightly more QALYs compared with the mindfulness group. The fact that most cost-effect pairs are situated in the southwest quadrant of the cost-effectiveness plane leads to a downward sloping CEAC. This means that there is a negative relationship between the value we assign to a QALY (i.e. the threshold) and the likelihood that MGT is cost-effective. At a threshold value of $€ 24$ 691/QALY, MGT has a $67 \%$ probability to be cost-effective from a societal perspective compared with TAU (Fig. 1).

It is important to consider that the duration of the trial was only 8 weeks, which is too short to observe the long-term effects of the intervention. In a future study, it would be interesting to find out whether the intervention remains cost saving or not with longterm follow-up data, especially as individuals with recurrent depression have a high risk of repeated depressive relapse or recurrence with rates in the range of $50-80 \% .{ }^{5}$ It has been shown that mindfulness-based cognitive therapy is an effective intervention for relapse prevention in patients with recurrent depressive disorder compared with TAU and placebo. ${ }^{28}$ If this holds true in the current Swedish setting, MGT can be expected to be even more cost-effective in the long-run.

\section{Comparison with findings from other studies}

It is difficult to compare health economic outcomes from one study with another for mindfulness therapy because of non-standardisation of methodologies and comparison groups. ${ }^{29}$ Nonetheless, such a comparison would be beneficial for policymakers in order to make informed choices considering all the factors associated with outcomes. Both Kuyken et $a l^{5}$ and Morriss et al found that MBT was not better or worse in terms of costs or effects for patients with depression in the UK compared with antidepressive medication and usual care, respectively. Besides the settings being different, both studies had a longer follow-up, 24-month and 18-month respectively, compared with the current study. Shawyer et $a l^{7}$ found that MBT was a cost-effective option compared with 'active monitoring for depressive relapse' for a population of currently non-depressed adults with a history of major depressive episodes in Australia. ${ }^{7}$

\section{Interpretation of our findings from the subgroup and sensitivity analyses}

Our base case results hold in the subgroup and sensitivity analyses. In a few cases, the base case finding of significant difference in cost is lost (stratifying for gender and removing outliers in QALY gain, see Table 3). Especially for men, this is likely an effect of the concurrent reduction in sample size. Indications are also that in the analyses of women and QALY outliers, MGT is the preferred treatment as TAU would not be considered cost-effective compared with MGT. The subgroup and sensitivity analyses thus suggest that the cost-saving characteristics of MGT in the base case estimates hold, and that MGT is, at the very least, not inferior to TAU.

\section{Strengths and limitations}

To our knowledge, this is the first economic evaluation of an RCT performed in a PHC setting where MBT has been compared with TAU. In addition, this was a pragmatic study, resembling the actual healthcare practice in PHCs for participants with depression, anxiety, stress and adjustment disorders (i.e. we had an active control group).

The study also has some limitations. We used the UK tariff to calculate QALYs for the Swedish population. In the absence of an equivalent for the Swedish population for EQ-5D-5L, the use of the UK tariff was deemed appropriate. The RCT was underpowered to detect cost and QALY differences because the power calculation was based on depression- and anxiety-related outcomes. Cost data usually follow a highly skewed distribution, implying a need for larger sample sizes in cost-effectiveness studies as compared with effectiveness studies. ${ }^{30}$ Self-reported data were collected on medication use, which is prone to recall bias.

The societal perspective included only productivity in addition to the healthcare perspective. Other societal costs, for example the participants' time and travel costs were not included because of lack of data. This is also true for the healthcare perspective, for example, general practitioner visits outside the treatment as well as overhead costs were not included. Moreover, data was lacking on exactly when the participants returned to work and/or went on sick leave during this 8-week period as the questionnaire was used at baseline and the end of the trial. Therefore, we assumed 1 week of productivity gain/loss, which is probably an underestimation.

The duration of the study was only 8 weeks and thus caution is advised when extrapolating the cost-effectiveness of MGT in the long run. Finally, caution is also needed when generalising the results of this study to other contexts. When using the MGT in a different setting the population characteristics as well as social, political, cultural and healthcare services in which the programme will be implemented and used, need to be taken into consideration.

In any future studies, instead of self-reported information on healthcare use, a register-based study would provide actual estimates of the patients' healthcare use and thus can validate the self-reported information.

In conclusion, mindfulness-based therapies can be performed on a group basis and can also be continued at home without an instructor, which makes it an attractive alternative to individual CBT. We found that MGT is cost saving compared with TAU. However, further studies will need to examine long-term costeffectiveness before any final recommendations can be given.

Sanjib Saha, PhD, Postdoctoral Researcher, Health Economics Unit, Department of Clinical Sciences (Malmö), Lund University, Sweden; Johan Jarl, PhD, Associate Professor, Health Economics Unit, Department of Clinical Sciences (Malmö), Lund University, Sweden; Ulf-G Gerdtham, PhD, Professor, Health Economics Unit, Department of Clinical Sciences (Malmö), Department of Economics, Centre for Economic Demography and Center for Primary Health Care Research, Lund University, Sweden; Kristina Sundquist, MD, PhD, Professor, Center for Primary Health Care Research, Lund University, Sweden; Department of Family Medicine and Community Health, Icahn School of Medicine at Mount Sinai, USA; and Center for Community-based Healthcare Research and Education, Shimane University, Japan; Jan Sundquist, MD, PhD, Professor, Center for Primary Health Care Research, Lund University, Sweden; Department of Family Medicine and Community Health, Icahn School of Medicine at Mount Sinai, USA; and Center for Community-based Healthcare Research and Education, Shimane University, Japan

Correspondence: Sanjib Saha, Health Economics Unit, Department of Clinical Sciences (Malmö), Lund University, Medicon village, Scheelevägen 2, SE-22381, Lund, Sweden. Email: sanjib.saha@med.lu.se

First received 18 Dec 2017, final revision 21 Sep 2018, accepted 9 Oct 2018

\section{Supplementary material}

Supplementary material is available online at https://doi.org/10.1192/bjp.2018.247.

\section{Funding}

J.S. and K.S. received funding from the Swedish Research Council (VR 2014-10134 and VR 201802400), ALF funding from Region Skåne and The Swedish Research Council for Health, Working 
Life and Welfare (in Swedish: Forte). The Health Economics Program at Lund University receives ALF funding from Region Skåne (Gerdtham) (ALF; Dnr F:2014/354). The funding agencies had no role in the design and conduct of the study, nor in the collection, analysis and interpretation of the data or the preparation, review or approval of the manuscript.

\section{Acknowledgements}

We are grateful to the participating 16 primary healthcare centres and the 30 mindfulness instructors performing the MGT.

\section{References}

1 Socialstyrelsen. Nationell utvärdering 2013 - Vård och Insatser vid Depression, Ångest och Schizofreni Rekommendationer, Bedömningar och Sammanfattning [The National Board of Health and Welfare. National evaluation, 2013 - care and interventions for depression, anxiety and schizophrenia, recommendations, estimates and summary]. Socialstyrelsen, 2013.

2 Morgan D. Mindfulness-based cognitive therapy for depression: a new approach to preventing relapse. Psychother Res 2003; 13: 123-5.

3 Sundquist J, Lilja A, Palmer K, Memon AA, Wang X, Johansson LM, et al. Mindfulness group therapy in primary care patients with depression, anxiety and stress and adjustment disorders: randomised controlled trial. $\mathrm{Br} J$ Psychiatry 2015; 206: 128-35.

4 Sundquist J, Palmer K, Johansson LM, Sundquist K. The effect of mindfulness group therapy on a broad range of psychiatric symptoms: a randomised controlled trial in primary health care. Eur Psychiatry 2017; 43: 19-27.

5 Kuyken W, Hayes R, Barrett B, Byng R, Dalgleish T, Kessler D, et al. The effectiveness and cost-effectiveness of mindfulness-based cognitive therapy compared with maintenance antidepressant treatment in the prevention of depressive relapse/recurrence: results of a randomised controlled trial (the PREVENT study). Health Technol Assess 2015; 19: 1-124.

6 Morriss R, Garland A, Nixon N, Guo B, James M, Kaylor-Hughes C, et al. Efficacy and cost-effectiveness of a specialist depression service versus usual specialist mental health care to manage persistent depression: a randomised controlled trial. Lancet Psychiatry 2016; 3: 821-31.

7 Shawyer F, Enticott JC, Ozmen M, Inder B, Meadows GN. Mindfulness-based cognitive therapy for recurrent major depression: a 'best buy' for health care? Aust N Z J Psychiatry 2016; 50: 1001-13.

8 Kabat-Zinn J, Lipworth L, Burncy R, Sellers W. Four-year follow-up of a meditation-based program for the self-regulation of chronic pain: treatment outcomes and compliance. Clin J Pain 1986; 2: 159-774.

9 Kabat-Zinn J, Massion AO, Kristeller J, Peterson LG, Fletcher KE, Pbert L, et al. Effectiveness of a meditation-based stress reduction program in the treatment of anxiety disorders. Am J Psychiatry 1992; 149: 936-43.

10 Herdman M, Gudex C, Lloyd A, Janssen M, Kind P, Parkin D, et al. Development and preliminary testing of the new five-level version of EQ-5D (EQ-5D-5L). Qual Life Res 2011; 20(10): 1727-36.

11 Devlin NJ, Shah KK, Feng Y, Mulhern B, van Hout B. Valuing health-related quality of life: an EQ-5D-5L value set for England. Health Econ 2018; 27: 7-22.
12 Manca A, Hawkins N, Sculpher MJ. Estimating mean QALYs in trial-based costeffectiveness analysis: the importance of controlling for baseline utility. Health Econ 2005; 14: 487-96.

13 Drummond MF, Sculpher MJ, Torrance GW, O'Brien BJ, Stoddart GL. Methods for the Economic Evaluation of Health Care Programmes. Oxford University Press, 2005.

14 Swedish Association of Local Authorities and Regions. Cost per person database for specialized care. Swedish Association of Local Authorities and Regions, no date (https://statva.skl.se/KPP somatik_publik.html).

15 Sveriges Riskbank. Exchange Rate. Sveriges Riskbank, no date (https://www. riksbank.se/en-gb/statistics/search-interest--exchange-rates/).

16 Weisbrod BA. The valuation of human capital. J Polit Econ 1961; 69: 425-36.

17 The Swedish National Mediation Office. Genomsnitts/öner 2012. The Swedish National Mediation Office, 2012 (http://www.mi.se/files/PDF-er/ar_foreign/ eng_wage_differentials_2012.pdf).

18 Briggs $\mathrm{AH}$, Wonderling $\mathrm{DE}$, Mooney CZ. Pulling cost-effectiveness analysis up by its bootstraps: a non-parametric approach to confidence interval estimation. Health Econ 1997; 6: 327-40.

19 Efron B, Tibshirani RJ. An Introduction to the Bootstrap. CRC Press, 1994.

20 Briggs A, Fenn P. Confidence intervals or surfaces? Uncertainty on the costeffectiveness plane. Health Econ 1998; 7: 723-40.

21 Fenwick E, O'Brien BJ, Briggs A. Cost-effectiveness acceptability curves-facts, fallacies and frequently asked questions. Health Econ 2004; 13: 405-15.

22 National Institute for Health and Care Excellence. Guide to the Methods of Technology Appraisal. NICE, 2004.

23 Husereau D, Drummond M, Petrou S, Carswell C, Moher D, Greenberg D, et al. Consolidated Health Economic Evaluation Reporting Standards (CHEERS) statement. Br Med J 2013; 346; f1049.

24 Lavori PW, Dawson R, Shera D. A multiple imputation strategy for clinical trials with truncation of patient data. Statist Med 1995; 14: 1913-25.

25 Rubin D. Multiple Imputation for Nonresponse in Surveys. Wiley \& Sons, 1987.

26 Frew E. Applied Methods of Cost-Benefit Analysis in Health Care. Oxford University Press, 2010.

27 Lejtzen N, Sundquist J, Sundquist K, Li X. Depression and anxiety in Swedish primary health care: prevalence, incidence, and risk factors. Eur Arch Psychiatry Clin Neurosci 2014; 264: 235-45.

28 Piet J, Hougaard E. The effect of mindfulness-based cognitive therapy for prevention of relapse in recurrent major depressive disorder: a systematic review and meta-analysis. Clin Psychol Rev 2011; 31: 1032-40.

29 Edwards RT, Bryning L, Crane R. Design of economic evaluations of mindfulness-based interventions: ten methodological questions of which to be mindful. Mindfulness 2015; 6: 490-500.

30 Briggs A. Economic evaluation and clinical trials: size matters. BMJ 2000; 321: 1362-3. 